\title{
Nuevos registros del Pimpollo Tobiano Podiceps gallardoi (Podicipedidae) en Patagonia Chilena
}

\author{
New records of the Hooded Grebe Podiceps gallardoi \\ (Podicipedidae) in chilean Patagonia
}

Roberto Donoso ${ }^{1}$, Sebastián Saiter ${ }^{2}$, Ricardo Matus ${ }^{3}$ \& Alejandro Kusch ${ }^{4}$

El Pimpollo tobiano Podiceps gallardoi se describió como especie nueva para la ciencia en 1974 a partir de ejemplares capturados en la Patagonia Argentina (Rumboll, 1974) y desde entonces se ha tratado de descifrar la distribución estival $e$ invernal de la especie. En la actualidad se define su rango de distribución estival como el territorio de la Provincia de Santa Cruz, al este de la Cordillera de los Andes, que se caracteriza por presentar mesetas de altura por encima de los 800 $\mathrm{m}$ snm, con numerosas lagunas, extendidas entre los $47^{\circ} \mathrm{S}$ y $51^{\circ} \mathrm{S}$ (Beltrán et al. 1992; Roesler et al. 2012a). En periodo invernal, las aves se mueven hacia ambientes de menor elevación en la costa atlántica, principalmente entre la confluencia de los ríos Chico y Santa Cruz en el norte y la desembocadura del río Gallegos en el sur, entre los meses de abril y agosto llegando excepcionalmente a la Bahía de San Julián (Imberti et al. 2004). Registros fuera de este rango incluyen cuatro ejemplares observados en septiembre (18/09/2009), en una laguna a $28 \mathrm{~km}$ al sur de la ciudad de Río Gallegos (Ea. 3 de Enero, 51 $52^{\circ}$ S, $69^{\circ} 26^{\prime}$ O, R. Donoso \& A. Kusch, datos no publicados). Prospecciones en la zona de fiordos $y$ canales patagónicos durante el periodo invernal no registraron la especie (Imberti, 2005).

El Pimpollo tobiano ha visto decrecer su población drásticamente, hasta en un $80 \%$ en algunas mesetas en los últimos 25 años (Roesler et al. 2012a) y últimamente los trabajos de conservación e investigación con esta especie se han concentrado en contrarrestar su carácter de especie altamente amenazada por depredación de visones y gaviotas, y la introducción de salmónidos en las lagunas que habita (Roesler et al. 2012b), lo que sumado a un rango de distribución restringido, llevaron a categorizar la especie como críticamente amenazada (IUCN, 2015).

En el extremo sur de la Patagonia Chilena (51-53 S), región de Magallanes, se han realizado registros esporádicos. El 5 de febrero de 1997 en Tierra del Fuego, St. Pierre \& Davis (1998) entre otros. Sin embargo, se ha aceptado sólo un registro con evidencias en Tierra del Fuego (Matus, 1998 con material fotográfico del autor), ocurrido en la laguna Santa María (26/10/1997). Aunque Narosky \& Babarskas (2001) lo mencionan como habitante de Tierra del Fuego Chilena, ni ellos ni otros autores aportan más datos y no se han dado a conocer nuevos avistamientos en la isla (K. Roesler com. pers.).

Estas observaciones le dieron el carácter de visitante accidental en el sur de Chile según varios autores (Couve \& Vidal, 2003; Jaramillo, 2003). Jaramillo menciona que es posible que sea una especie más regular de lo que se considera, probablemente debido a que también existen otros registros inéditos de observadores confiables en el Parque Nacional Torres del Paine en los años 90's

\footnotetext{
1 Patagonia Journeys. patagoniajourneys@gmail.com

2 Agrupación Ecológica Patagónica. sebastian.saiter.v@gmail.com

$3 \quad$ Natura Patagonia. rmatusn@gmail.com

$4 \quad$ Wildlife Conservation Society-Chile. akusch@wcs.org $\square$
} 
(P. Harrison com. pers a S. Imberti). Roesler et al. (2012a) lo consideran como visitante marginal en Patagonia Chilena. En esta nota presentamos una serie de observaciones inéditas en Magallanes ocurridas entre octubre de 2013 y abril de 2015 , en dos localidades distintas de la porción continental de la Patagonia Chilena.

Entre el mes de octubre de 2013 y enero de 2014 se registraron en la laguna Kampenaike (52 42'30' ' S, 7053'50' O) uno a dos ejemplares (Tabla 1). Este sector se visitó en busca del Pimpollo Tobiano en base a registros sin confirmar de años anteriores (J. Cárcamo com. pers.). En el periodo estival siguiente se supo por parte de personal de CONAF que en noviembre del 2014 se realizó un registro sin confirmar al interior del Parque Nacional Torres del Paine. Entre diciembre de 2014 y abril de 2015 , se observaron en laguna de los Blanquillos (51 00 '16" S, 7248'35' O) en este parque nacional, tres a cuatro ejemplares (Tabla 1). En este sitio se observaron pares de pimpollos tobianos en comportamiento agonístico (ver Erize, 1983 para mas detalles sobre la especie). Se observaron persecuciones, algunas con el plumaje del manto erizado pero no se observó robo de material ni plataformas de nido. Estos registros se distancian respectivamente a $100 \mathrm{~km} \mathrm{y} 230 \mathrm{~km}$ de la meseta de las Vizcachas que corresponden al límite sur de la distribución del pimpollo (Beltrán et al., 1992; Roesler et al., 2012a).

Las observaciones de aves poco conocidas para la zona austral de Chile ocurren principalmente en el ecosistema de estepa

Tabla 1. Registros de Pimpollo tobiano, Podiceps gallardoi, en Magallanes entre los años 2013 y 2015. Todas las observaciones poseen fotografías de respaldo.

\begin{tabular}{cc}
\hline Localidad & Fechas (no. ejemplares) \\
\hline Lag. Kampenaike & 12 y 19 Oct., 2013 (1 y 2) \\
14 y 17 Dic., 2013 (1) \\
04 Ene., $2014(1)$ \\
27 Dic., $2014(4)$ \\
11 Ene., $2015(4)$ \\
Lag. Blanquillos & 03 Feb., $2015(4)$ \\
& 12 y 24 Mar., 2015 (4) \\
4,6 y 18 Abr., 2015 (4 y 5)
\end{tabular}

patagónica. El conocimiento del estatus de muchas especies es incipiente y puede ocurrir que aquellas consideradas raras son en realidad frecuentes aunque en bajos números poblacionales, o que aquellas aves consideradas migrantes pueden no ser estrictos y tener también movimientos regionales (Matus \& Jaramillo, 2008). Las observaciones del Pimpollo Tobiano presentadas son de pocos ejemplares, sin embargo su permanencia durante dos temporadas reproductivas y el hecho de tratarse de una especie críticamente amenazada, resulta propicia para apoyar la idea de mantener prospecciones ornitológicas sistemáticas en la región y validar cambios en la distribución de especies en los extremos de sus rangos de distribución conocidos como ha sido propuesto para otras especies (Vuilleumier, 1994) o evaluar la distribución interanual en condiciones climáticas favorables.

Los ejemplares observados en los últimos años resultan relevantes porque son los primeros que han sido registrados con una permanencia de un periodo estival completo, durante dos temporadas seguidas, en dos nuevas localidades. Por el momento el estatus del Pimpollo Tobiano en nuestro país puede mantenerse como hasta el presente y de registrarse más ejemplares y/o comportamiento reproductivo se deberá evaluar su residencia en Magallanes.

\section{AGRADECIMIENTOS}

Agradecemos a la estación INIA Kampenaike por permitir el acceso a su predio y a José Díaz T. y Fernando Díaz S. por facilitarnos sus registros. En especial a Jaime Cárcamo por discutir registros históricos y a Kini Roesler por sus valiosos comentarios que ayudaron a mejorar el manuscrito.

\section{LITERATURA CITADA}

Beltrán, J., Bertonatti, C., Johnson, A., Serret, A., \& Sutton, P. (1992). Actualizaciones sobre la distribución, biología y estado de conservación del Macá Tobiano (Podiceps gallardoi). Hornero, 13, 193-199.

Couve, E., \& Vidal, C. (2003). Aves de Patagonia, Tierra del Fuego y Península Antártica. Punta Arenas, Chile: Editorial Fantástico Sur 
Birding Ltd.

Erize, F. (1983). Observaciones sobre el Macá tobiano. Hornero Número Extraordinario, 12, 256-268.

Imberti, S., Sturzenbaum, S., \& McNamara, M. (2004). Actualización de la distribución invernal del Macá Tobiano (Podiceps gallardoi) y notas sobre su problemática de conservación. Hornero, 19, 83-89.

Imberti, S. (2005) Distribución otoñal de aves marinas y terrestres en los Canales Chilenos. Anales del Instituto de la Patagonia, 33, 21-30.

IUCN. (2015). The IUCN Red List of Threatened Species. Version 2015.2. www.iucnredlist. org. Descargado el 14 de julio 2015.

Jaramillo, A. (2003). Birds of Chile. Lynx Edicions.

Matus, R. (1998). Presencia accidental de Corvus splendens (Aves, Corvidae) y nuevos registros de aves raras en Magallanes: Rollandia gallardoi y Eremobius phoenicurus. Anales Instituto de la Patagonia, 26, 137-139.

Matus, R., \& Jaramillo, A. (2008). Range extensions and vagrant bird species in the XII Region of Magallanes, Chile. Cotinga, 30, 34-40.

Narosky, T., \& Babarskas, M. (2001). Aves de la
Patagonia. Vázquez Mazzini Editores.

Roesler, I., Imberti, S., Casañas, H., Mahler, B., \& Reboreda, J. C. (2012a). Hooded Grebe Podiceps gallardoi population decreased by eighty per cent in the last twenty-five years. Bird Conservation International, 22(4), 371-382.

Roesler, I., Imberti, S., Casañas, H., \& Volpe, N. (2012b). A new threat for the globally Endangered Hooded Grebe Podiceps gallardoi: the American mink Neovison vison. Bird Conservation International, 22(4), 383-388.

Rumboll, M. (1974). Una nueva especie de Macá (Podicipitidae). Revista del Museo Argentino de Ciencias Naturales "Bernardino Rivadavia", 4, 33-35.

St. Pierre, P., \& Davis, M. (1998). Observaciones ornitológicas en el Monumento Natural Laguna de los Cisnes, Tierra del Fuego: nuevo registro de Pimpollo tobiano (Podiceps gallardoi) en Chile. Boletín Chileno de Ornitología, 5, 28-29.

Vuilleumier, F. (1994). Nidificación y status de Phrygilus fruticeti (Aves: Emberizidae) en la Patagonia Chilena: un ejemplo del fenómeno de límite de la especie? Rev. Chil. Hist. Nat., 67, 299-307. 
R. DONOSO et al. 